\title{
Mesenchymal-epithelial Transition and Tumor Vascular Remodeling in Eribulin Chemotherapy for Breast Cancer
}

\author{
SHINICHIRO KASHIWAGI ${ }^{1}$, YUKA ASANO ${ }^{1}$, WATARU GOTO ${ }^{1}$, KOJI TAKADA $^{1}$, KATSUYUKI TAKAHASHI $^{2}$, \\ TAKAHARU HATANO ${ }^{3}$, SAYAKA TANAKA $^{4}$, TSUTOMU TAKASHIMA ${ }^{1}$, SHUHEI TOMITA ${ }^{2}$, \\ HISASHI MOTOMURA ${ }^{3}$, MASAHIKO OHSAWA ${ }^{4}$, KOSEI HIRAKAWA ${ }^{1}$ and MASAICHI OHIRA ${ }^{1}$ \\ Departments of ${ }^{1}$ Surgical Oncology, ${ }^{2}$ Pharmacology, ${ }^{3}$ Plastic and Reconstructive Surgery, and \\ ${ }^{4}$ Diagnostic Pathology, Osaka City University Graduate School of Medicine, Osaka, Japan
}

\begin{abstract}
Background/Aim: Eribulin mesylate (eribulin) is currently used for the treatment of locally advanced or metastatic breast cancer (MBC). It is a cytotoxic agent with unique mechanisms that suppress the epithelialmesenchymal transition (EMT) of cancer cells and promote tumor vascular remodeling. In this study, we investigated the expression of markers for EMT and hypoxia in sets of clinical specimens collected before and after eribulin treatment to verify its unique mechanisms. Patients and Methods: The expression of markers for EMT and cellular hypoxia [E-cadherin, $N$-cadherin, vimentin, and carbonic anhydrase 9 (CA9)] was examined immunohistochemically in MBC tissues collected from 20 patients before and after chemotherapy with either eribulin $(n=10)$ or paclitaxel $(n=10)$. Results: An increase of E-cadherin and decrease of CA9 expression were observed in MBC tissues from patients with objective clinical responses to eribulin treatment. Patients with E-cadherin-positive conversion and CA9-negative conversion had significantly higher response rates ( $p=0.004$ and $p=0.024$, respectively) and prolonged time to treatment failure $(p=0.018$ and $p=0.038$, respectively) than patients without changes in marker expression. Conclusion: Expression of EMT and hypoxia markers in clinical samples from patients with $M B C$ was suppressed by eribulin treatment. The results provide additional clinical data on improved survival of patients treated with eribulin and the mechanism of response.

Several synthetic compounds that interact with microtubules
\end{abstract}

Correspondence to: Dr. Shinichiro Kashiwagi, Osaka City University Graduate School of Medicine, 1-4-3 Asahi-machi, Abeno-ku, Osaka 545-8585, Japan. Tel: +81 666453838, Fax: +81 666466450, e-mail: spqv9ke9@view.ocn.ne.jp

Key Words: Eribulin mesylate, epithelial-mesenchymal transition, tumor vascular remodeling, locally advanced breast cancer, metastatic breast cancer, E-cadherin. have demonstrated a clinical utility against malignancies including breast cancer. Eribulin mesylate (eribulin) is a new synthetic derivative of halichondrin $\mathrm{B}$, which was originally isolated from Halichondria okadai (1). Eribulin suppresses cell division by inhibiting microtubule extension through a mechanism that differs from that of other antimitotic drugs such as taxane and vinca alkaloids (2-5). It binds to microtubule ends and inhibits microtubule polymerization (6, 7). Taxane binds extensively inside microtubules and suppresses shortening of microtubules by depolymerization. Vinca alkaloids bind to the external surface of microtubules and suppress both microtubule polymerization and depolymerization. Consequently, the anticancer effects differ among these agents. For example, in a phase III trial of eribulin (Eisai Metastatic Breast Cancer Study Assessing Physician's Choice versus E7389, EMBRACE), significant prolongation of overall survival was observed in patients with locally advanced or metastatic breast cancer (MBC) after eribulin treatment even without improvement of disease-free survival (6). This result was partially explained by a decrease in the occurrence of new metastatic lesions after eribulin therapy, an effect that has not been demonstrated by other drugs. However, the precise mechanism for this clinical benefit has not yet been elucidated.

Some of the unique anticancer effects of eribulin have been revealed by experimental studies of cancer cells and tumor tissues. These effects include suppression of epithelial-mesenchymal transition (EMT) in cancer cells and promotion of vascular remodeling in tumors $(7,8)$. EMT is observed when cancer metastasizes, and promotes cancer infiltration and metastasis by facilitating the ability of cancer cells to move and breakdown the extracellular matrix. Cancer cells with induced EMT are known to acquire treatment resistance and enhanced properties as cancer stem cells (9-11). The expression of E-cadherin, an adhesion molecule expressed by epithelial cells, declines during EMT as the expression of mesenchymal markers, such as $\mathrm{N}$ cadherin and vimentin, increases (12-14). The epithelial and 
mesenchymal phenotypes of cancer cells can thus be distinguished by the relative expression of these markers.

Vascular remodeling involves the restoration of tumor vasculature in the vicinity of cancer cells. Most tumors have hypoxic regions because of aggressive cell proliferation that exceeds the ability to develop a network of blood vessels to nourish the cancer cells. Thus, adequate vascularization is important for tumor survival. Cancer cells are also known to acquire a high metastatic potential upon exposure to hypoxic environments $(15,16)$. Therefore, vascular remodeling might inhibit aggressive metastasis by enhancement of the hypoxic state. Eribulin was reported to improve blood circulation in the central region of subcutaneous tumor tissue implants in a mouse model (7). Hypoxia induces expression of carbonic anhydrase 9 (CA9), which is mediated by hypoxia-inducible factor-1 (HIF-1) $(17,18)$. The expression of CA9 in cancer cells indicates a hypoxic region within solid cancer tissue, and may serve as a marker of hypoxia (17).

To date, few studies have reported the effects of eribulin on cancer cell characteristics or tumor vasculature in clinical specimens. In this study, differences in the expression of EMT and hypoxia markers were determined by immunohistochemical staining of MBC tissues obtained from patients before and after drug administration. The objective of this study was to confirm EMT suppression and vascular remodeling induced by eribulin in a clinical setting.

\section{Patients and Methods}

Patient background. Eribulin chemotherapy was administered to 52 patients with locally advanced breast cancer or MBC at Osaka City University Hospital from August 2011 to June 2013. Cancer tissue samples were collected from 10 patients before and after treatment. The median observation period was 431 days (range $=50-650$ days) for eribulin treatment, and 420 days (range $=37-650$ days) for paclitaxel treatment. The objective response rate (ORR) [complete response $(\mathrm{CR})+$ partial response $(\mathrm{PR})]$, clinical benefit rate $(\mathrm{CBR})$ $(\mathrm{CR}+\mathrm{PR}+$ stable disease $(\mathrm{SD})>24$ weeks], disease control rate (DCR) $(\mathrm{CR}+\mathrm{PR}+\mathrm{SD})$, overall survival (OS), time to treatment failure (TTF) and progression-free survival (PFS) were calculated regarding the efficacy of this regimen. The chemotherapy regimen included $1.4 \mathrm{mg} / \mathrm{m}^{2}$ eribulin administered intravenously to outpatients on days 1 and 8 of a 21-day course of treatment (6), and administration was repeated until confirmation of progressive disease (PD). Cancer tissue samples collected from 10 age-matched paclitaxel-treated patients ( $80 \mathrm{mg} / \mathrm{m}^{2}$ weekly) with maximal clinical responses were used as controls for immunohistochemistry.

Pathological diagnoses were performed by experienced pathologists using hematoxylin and eosin-stained tissue samples obtained either during surgery or by core needle biopsy. This study conformed to the provisions of the Declaration of Helsinki 1995. All patients were informed of the investigational nature of this study and provided their written informed consent. The study protocol was approved by the Ethics Committee of Osaka City University (\#926). Immunohistochemistry. Immunohistochemical staining was performed as previously described $(19,20)$. Tissue specimens were fixed in $10 \%$ formaldehyde and embedded in paraffin. Sections were cut at $4-\mu \mathrm{m}$ thicknesses, mounted on glass slides, deparaffinized in xylene, placed in Target Retrieval Solution (Dako, Carpinteria, CA, USA), and heated for $20 \mathrm{~min}$ in an autoclave at $105^{\circ} \mathrm{C}$ and $0.4 \mathrm{~kg} / \mathrm{m}^{2}$. The specimens were then immersed in methanol containing $3 \%$ hydrogen peroxide for 15 min to block endogenous peroxidase activity, followed by incubation in $10 \%$ normal goat or rabbit serum to block nonspecific binding.

Primary monoclonal antibodies against E-cadherin (clone $\mathrm{NCH}-$ 38, 1:200 dilution; Dako), N-cadherin (clone 6G11, 1:50 dilution; Dako), Vimentin (clone Vim 3B4, 1:500 dilution; Dako), and CA9 (clone M75, 1:1000 dilution; Novus Biologicals, CO, USA) were used. The tissue sections were incubated with each antibody for 70 min at room temperature or overnight at $4{ }^{\circ} \mathrm{C}$, followed by incubation with horseradish peroxidase-conjugated anti-rabbit or anti-mouse Ig polymer (Histofine $\mathrm{PO}^{\mathrm{TM}}$ kit; Nichirei, Tokyo, Japan) as the secondary antibody. The sections were subsequently treated with streptavidin-peroxidase reagent and incubated in phosphatebuffered saline containing diaminobenzidine and $1 \%$ hydrogen peroxide $(\mathrm{v} / \mathrm{v})$, followed by counterstaining with Mayer's hematoxylin. Positive and negative controls for each marker were used according to the supplier's data sheet.

Immunohistochemical staining was evaluated by two pathologists specialized in mammary gland pathology (STa and MOhs) and were blinded to the patient treatments. The cut-offs for positive staining were $>30 \%$ of cells with E-cadherin membrane staining (positive conversion) (20), $>10 \%$ of cells with $\mathrm{N}$-cadherin or vimentin cytoplasmic staining (21), and $>10 \%$ of cells with CA9 membrane staining (negative conversion) (22). For quantitative analysis, specimens were evaluated before and after eribulin treatment. Cases with an increase in biomarker expression were defined as positive conversion, and those with decreases were defined as negative conversion.

Statistical analysis. Statistical analysis was performed using the SPSS ${ }^{\circledR}$ version 19.0 statistical software (IBM, Armonk, NY, USA). Categorical data are represented as numbers and percentages, and continuous data are represented as medians and range. The association between treatment efficacy and clinicopathological variables was analyzed using Fisher's exact test. The association of treatment with survival was analyzed using Kaplan-Meier plots and log-rank tests. In all analyses, $p<0.05$ was considered significant. Cutoff values for the biomarkers included in this study were chosen before conducting the statistical analysis.

\section{Results}

Eribulin- and paclitaxel-treated patients with investigated samples. Demographical data of patients who underwent chemotherapy for $\mathrm{MBC}$, and 10 eribulin- and paclitaxeltreated patients with investigated samples are summarized in Table I. There were no significance differences in patient backgrounds for both chemotherapies. In 11 eribulin-treated patients, it was possible to excise the lesion after treatment. One patient achieved CR. PR was achieved by five patients (50.0\%). SD for 24 months (long SD) occurred in one patient $(10.0 \%)$, SD in one patient $(10.0 \%)$, and PD in three patients $(30.0 \%)$. On the other hand, in 10 paclitaxel-treated patients, 
Table I. Demographical data of patients who underwent chemotherapy for locally advanced or metastatic breast cancer and eribulin-, and paclitaxeltreated patients with investigated samples.

\begin{tabular}{|c|c|c|c|c|}
\hline Parameters & & $\begin{array}{l}\text { Eribulin-treated patients } \\
\qquad(\mathrm{n}=10)\end{array}$ & $\begin{array}{l}\text { Paclitaxel-treated patients } \\
\qquad(\mathrm{n}=10)\end{array}$ & $p$-Value \\
\hline Age, years & Mean \pm SEM & $61.0 \pm 13.6$ & $62.0 \pm 12.3$ & 0.949 \\
\hline Degree of progress & Locally advanced/visceral metastases & $10(100.0 \%) / 0(0.0 \%)$ & $10(100.0 \%) / 0(0.0 \%)$ & 1.000 \\
\hline Previous treatment & $\begin{array}{l}\text { None/endocrine/chemo/ } \\
\text { anti-HER2 therapy }\end{array}$ & $\begin{array}{l}7(70.0 \%) / 0(0.0 \%) / \\
3(30.0 \%) / 0(0.0 \%)\end{array}$ & $\begin{array}{l}8(80.0 \%) / 0(0.0 \%) / \\
2(20.0 \%) / 0(0.0 \%)\end{array}$ & 0.606 \\
\hline Life threatening condition & Yes/no & $0(0.0 \%) / 10(100.0 \%)$ & $0(0.0 \%) / 10(100.0 \%)$ & 1.000 \\
\hline Nuclear grade & $1 / 2 / 3$ & $5(50.0 \%) / 1(10.0 \%) / 4(40.0 \%)$ & $4(40.0 \%) / 3(30.0 \%) / 3(30.0 \%)$ & 1.000 \\
\hline Estrogen receptor & Negative/positive & $5(50.0 \%) / 5(50.0 \%)$ & $5(50.0 \%) / 5(50.0 \%)$ & 1.000 \\
\hline Progesterone receptor & Negative/positive & $6(60.0 \%) / 4(40.0 \%)$ & $7(60.0 \%) / 3(30.0 \%)$ & 0.639 \\
\hline HER2 & Negative/positive & $10(100.0 \%) / 0(0.0 \%)$ & $10(100.0 \%) / 0(0.0 \%)$ & 1.000 \\
\hline $\operatorname{Ki67}(\geq 14 \%)$ & Negative/positive & $3(30.0 \%) / 70(70.0 \%)$ & $4(30.0 \%) / 60(60.0 \%)$ & 0.570 \\
\hline Intrinsic subtype & $\begin{array}{l}\text { Luminal A/luminal B/luminal } \\
\text { HER2/HER2-enriched/TNBC }\end{array}$ & $\begin{array}{c}3(30.0 \%) / 2(20.0 \%) / 0(0.0 \%) / \\
0(0.0 \%) / 5(50.0 \%)\end{array}$ & $\begin{array}{c}4(40.0 \%) / 1(10.0 \%) / 0(0.0 \%) / \\
0(0.0 \%) / 5(50.0 \%)\end{array}$ & 1.000 \\
\hline
\end{tabular}

HER2, Human epidermal growth factor receptor 2; TNBC, triple-negative breast cancer.

it was possible to excise the lesion after treatment. PR was achieved by five patients $(50.0 \%)$. Long SD occurred in one patient $(10.0 \%)$, SD in one patient $(10.0 \%)$, and PD in three patients $(30.0 \%)$. The efficacy of eribulin and paclitaxel chemotherapy is summarized in Table II.

Expression of EMT and hypoxia markers. Representative results of immunohistochemical staining are shown in Figure 1. Figure 2 shows the expression of the markers before and after treatment with the chemotherapeutic agents (Table III). E-Cadherin expression was found in $12-38 \%$ and $6-82 \%$ of cancer cells before and after eribulin treatment, respectively. After eribulin treatment, the E-cadherin expression status changed from negative to positive (positive conversion) in five patients, remained unchanged in four patients, and changed from positive to negative (negative conversion) in one patient (non-positive conversion). Prior to therapy, $\mathrm{N}$-cadherin and vimentin expression was positive in $7-70 \%$ and $7-72 \%$ of cancer cells, respectively. After therapy, positive expression was seen in $0-80 \%$ and $0-76 \%$ of cancer cells, respectively. Positive conversion of $\mathrm{N}$-cadherin was observed in one patient, and vimentin expression was seen in one patient. Negative conversion was seen in three patients, and biomarker expression was unchanged in six patients. CA9 expression was found in $3-82 \%$ of cancer cells prior to eribulin therapy and in $0-68 \%$ after therapy. Positive conversion of CA9 expression occurred in one patient, negative conversion was seen in four patients, and expression was unchanged in five patients. In contrast to the changes in protein expression after paclitaxel treatment, Ecadherin expression was unchanged in six patients. $\mathrm{N}$ cadherin was unchanged in eight patients, vimentin in six patients, and CA9 in seven patients.
Table II. Clinical effects of eribulin and paclitaxel chemotherapies on locally advanced or metastatic breast cancer.

\begin{tabular}{lcc}
\hline Measure & $\begin{array}{c}\text { Eribulin- } \\
\text { treated patients } \\
(\mathrm{n}=10), \mathrm{n}(\%)\end{array}$ & $\begin{array}{c}\text { Paclitaxel- } \\
\text { treated patients } \\
(\mathrm{n}=10), \mathrm{n}(\%)\end{array}$ \\
\hline ORR & $5(50.0 \%)$ & $5(50.0 \%)$ \\
CBR & $6(60.0 \%)$ & $6(60.0 \%)$ \\
DCR & $7(70.0 \%)$ & $7(70.0 \%)$ \\
PR & $5(50.0 \%)$ & $5(50.0 \%)$ \\
LSD & $1(10.0 \%)$ & $1(10.0 \%)$ \\
SD & $1(10.0 \%)$ & $1(10.0 \%)$ \\
PD & $3(30.0 \%)$ & $3(30.0 \%)$ \\
NE & $0(0.0 \%)$ & $0(0.0 \%)$ \\
$50 \%$ TTF (days) & 151 & 201 \\
$50 \%$ PFS (days) & 275 & 268 \\
$50 \%$ OS (days) & 422 & 386 \\
\hline
\end{tabular}

ORR: Objective response rate; CBR: clinical benefit rate; DCR: disease control rate: PR: partial response; LSD: stable disease >24 weeks; SD: stable disease; PD: progressive disease; NE; not evaluable; TTF: time to treatment failure; PFS: progression-free survival; OS: overall survival.

Anticancer responses (i.e. PR) to eribulin treatment were achieved by five patients (responders). All five responders demonstrated positive conversion to E-cadherin staining, Ecadherin expression was either unchanged or demonstrated negative conversion in the other five patients (nonresponders). Expression of all markers (N-cadherin, vimentin, and CA9) in tumors decreased significantly in the five responders. After treatment, E-cadherin expression was increased in the five control patients who responded to paclitaxel. However, the increase was smaller than that 


\section{A E-Cadherin-positive}

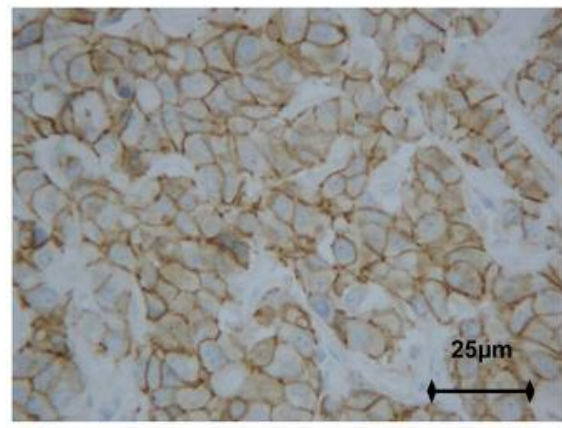

B N-Cadherin-positive

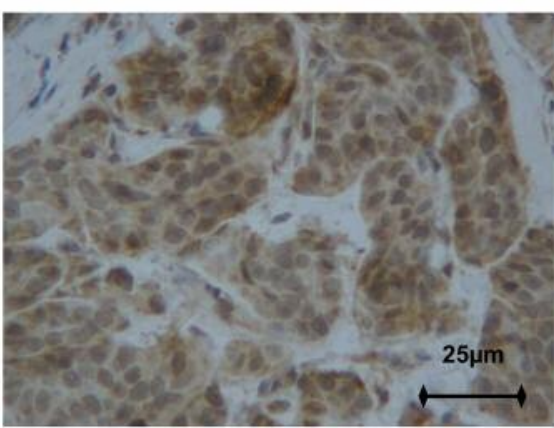

\section{Vimentin-positive}

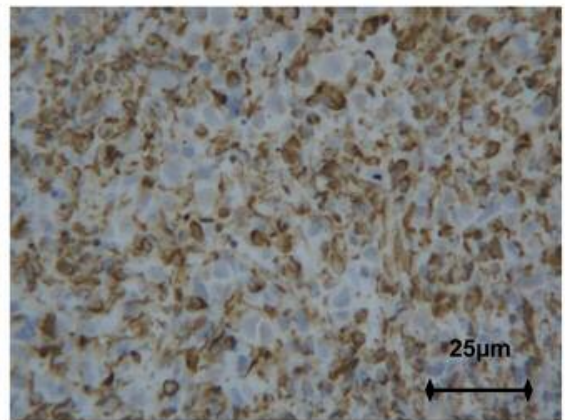

D CA9-positive

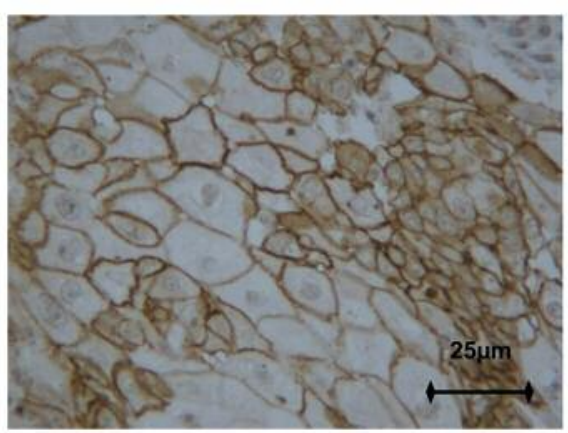

\section{E E-Cadherin-negative}

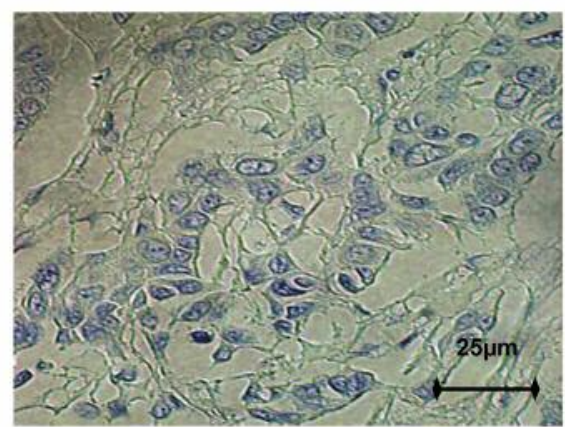

Figure 1. Immunohistochemical staining of breast cancer cells $(\times 400)$. A: E-Cadherin was primarily expressed at the cell membrane, with some staining visible the cytoplasm. $N$-Cadherin $(B)$ and vimentin $(C)$ were expressed in the cell cytoplasm, while carbonic anhydrase $9(C A 9)$ is primarily expressed at the cell membrane, with some staining visible in the cytoplasm (D). E: Example of negative E-cadherin staining.

Table III. Comparison of immunohistochemical quantification of all markers before and after treatment with eribulin or paclitaxel.

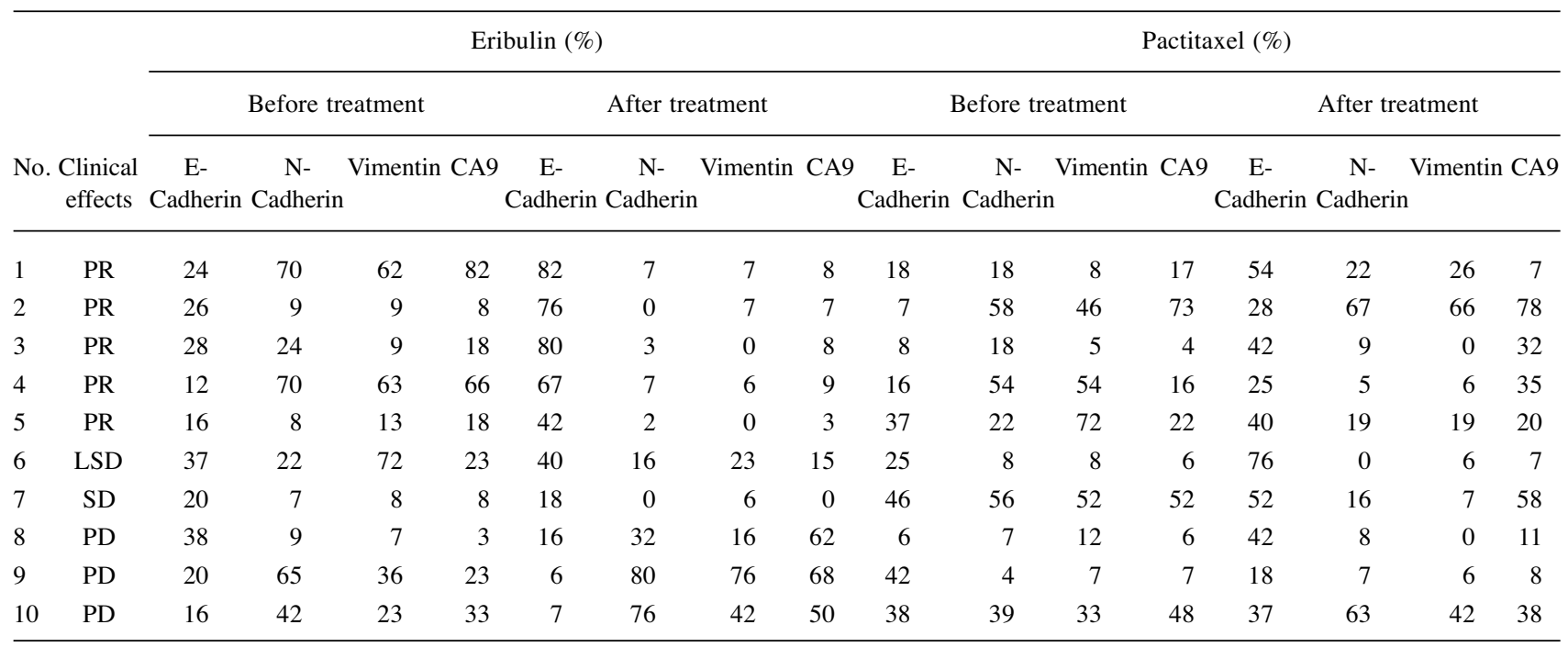

PR: Partial response; SD: stable disease; PD: progressive disease; LSD: long stable disease (SD >24 weeks); CA9: carbonic anhydrase 9. 


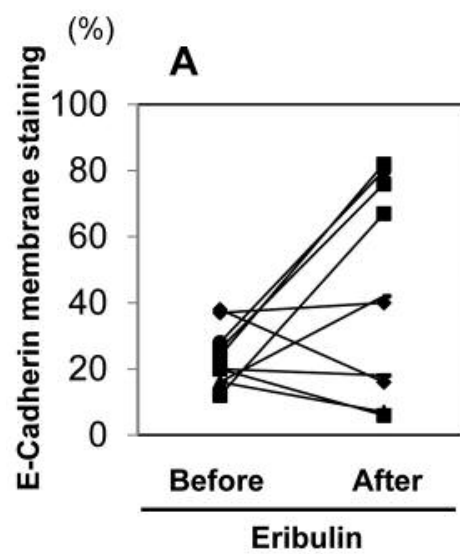

(\%)

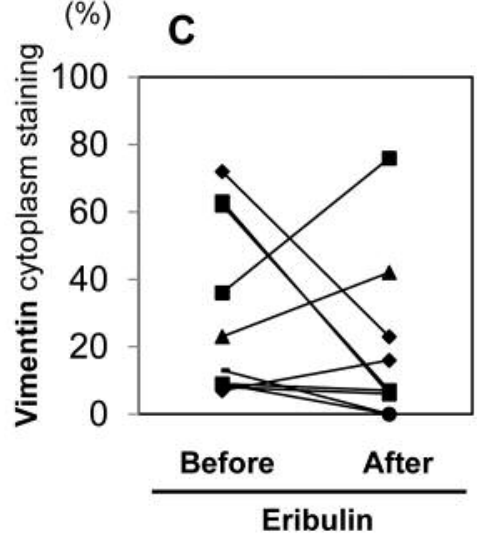

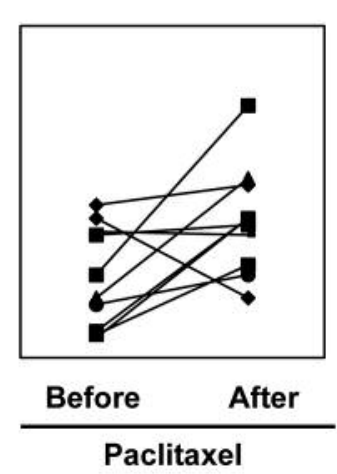

Paclitaxel

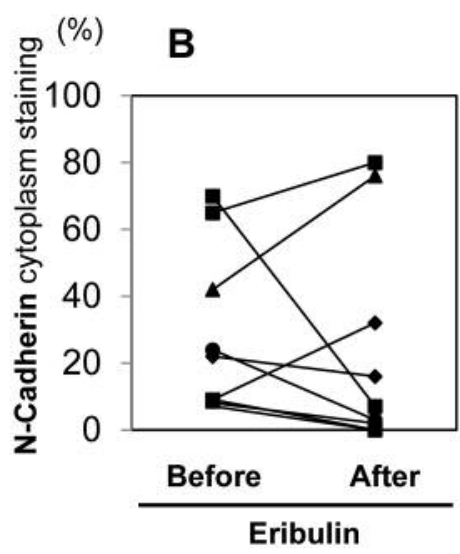

$(\%)$

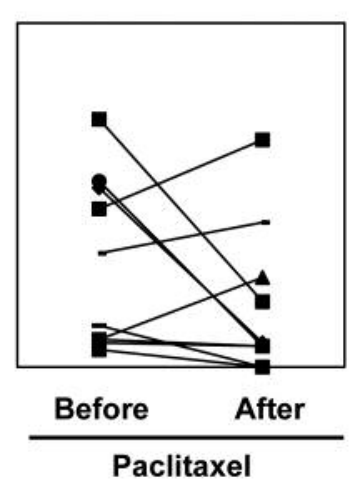

D

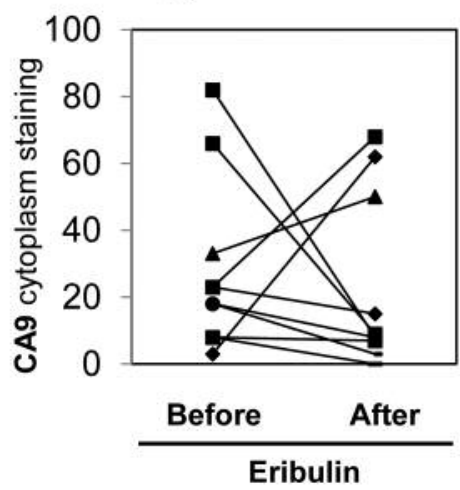

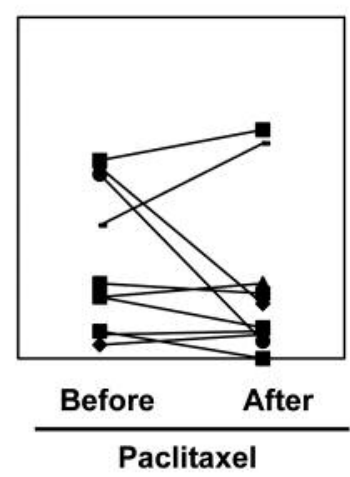

Paclitaxel

Figure 2. Comparison of marker expression before and after treatment with eribulin or paclitaxel. A: After eribulin treatment, the E-cadherin expression status changed from negative to positive (positive conversion) in five patients, remained unchanged in four patients, and changed from positive to negative (negative conversion) in one patient. B, C: Positive conversion of $N$-cadherin was observed in one patient (B), and vimentin expression in one patient $(C)$, negative conversion was seen in three patients, and their expression remained unchanged in six patients. D: Positive conversion of carbonic anhydrase 9 (CA9) expression occurred in one patient, negative conversion in four patients, and CA9 expression was unchanged in five patients. In contrast to the changes in protein expression after paclitaxel treatment, E-cadherin expression was unchanged in six patients. $N$-cadherin was unchanged in eight patients, vimentin in six patients, and CA9 in seven patients.

observed after eribulin therapy. No significant changes in $\mathrm{N}$-cadherin, vimentin, or CA9 expression were observed after effective paclitaxel treatment (Figure 3 and Table IV).

Patients with positive conversion of E-cadherin expression and negative conversion of CA9 expression after eribulin treatment achieved significantly higher response rates (RRs, $p=0.004$ and $p=0.024$, respectively) than patients without the corresponding conversions. Patients with decreased $\mathrm{N}$-cadherin and vimentin expression had a tendency for high RRs ( $p=0.083$ and $p=0.083$, respectively). In contrast, no significant correlations were found between marker expression and paclitaxel responses.

PFS was significantly prolonged in patients with tumors that showed positive conversion to E-cadherin $(p=0.041, \log$ rank test) (Figure 4). A significantly longer TTF was achieved in patients with tumors that showed positive conversion to Ecadherin ( $p=0.018, \log$-rank test) or negative conversion to CA9 ( $p=0.038, \log$-rank test) (Figure 5). There was no difference in OS of patients when stratified by expression and changes of EMT markers (Figure 6). In PFS, TTF, or OS of patients with expression of $\mathrm{N}$-cadherin and vimentin or changes in their expression, in many cases, it seems there was a difference, with a trend that was consistent with the model. However, the difference was not statistically significant.

\section{Discussion}

EMT of cells occurs during cancer progression (9-11), and expression of EMT markers in cancer cells has been associated with poor prognosis and acquisition of resistance 


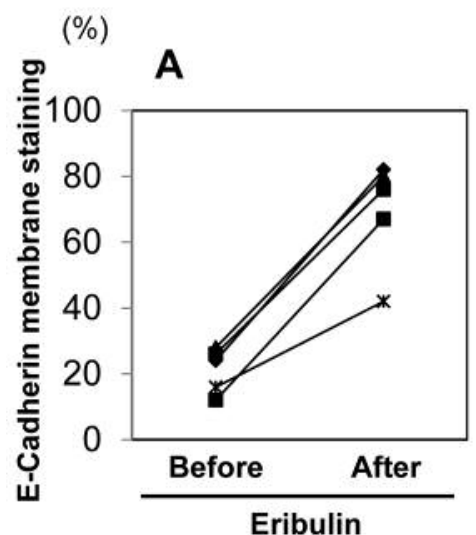

(\%)

C

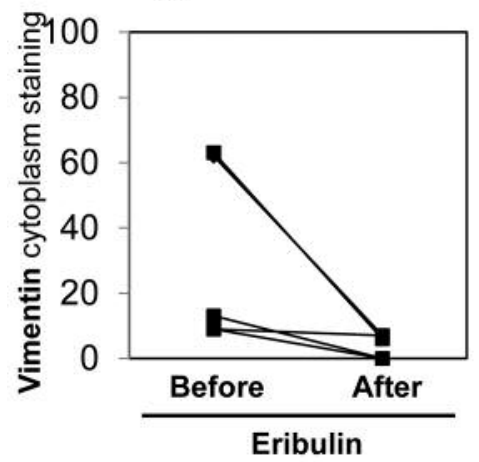

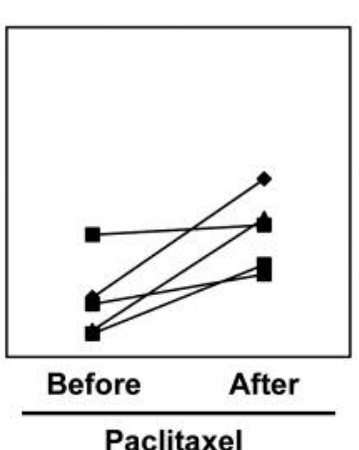

Paclitaxel

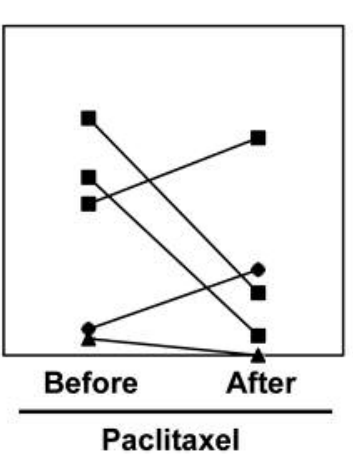

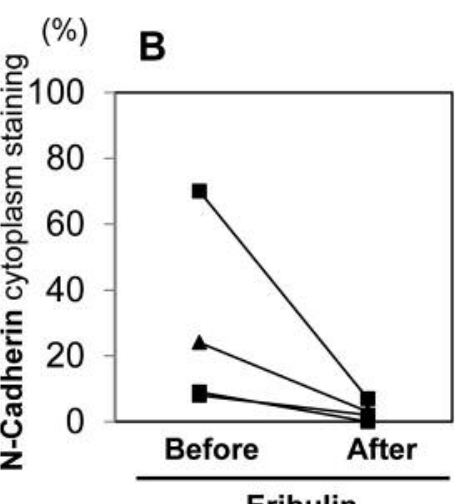

Eribulin

$(\%)$

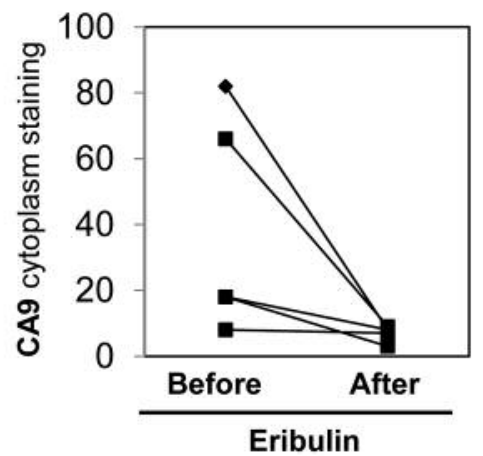

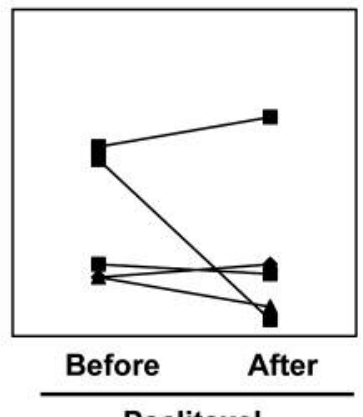

Paclitaxel

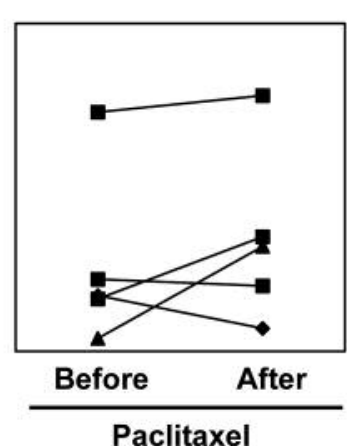

Figure 3. Comparison of marker expression before and after treatment with eribulin or paclitaxel in five patients with response patients (responders). All five responders to eribulin treatment demonstrated an E-cadherin-positive conversion (A). All five responders also demonstrated significant decreases in $N$-cadherin $(B)$, vimentin $(C)$, and carbonic anhydrase 9 (CA9) (D) expression of their tumors. In the five responders to paclitaxel treatment, E-cadherin expression was also increased after treatment. However, the extent was less than that seen after eribulin therapy. No significant changes in the expression of $\mathrm{N}$-cadherin, vimentin, or CA9 were observed after effective paclitaxel treatment.

Table IV. Correlation between responses to chemotherapy and marker expression in breast cancer tissues.

\begin{tabular}{|c|c|c|c|c|c|c|}
\hline \multirow[t]{2}{*}{ Parameters } & \multicolumn{3}{|c|}{ Eribulin } & \multicolumn{3}{|c|}{ Pactlitaxel } \\
\hline & Responders $(n=5)$ & Non-responders $(\mathrm{n}=5)$ & $p$-Value & Responders $(n=5)$ & Non-responders $(\mathrm{n}=5)$ & $p$-Value \\
\hline \multicolumn{7}{|l|}{ HR \& HER2 status } \\
\hline TNBC & $4(80 \%)$ & $3(60 \%)$ & & $3(60 \%)$ & $2(40 \%)$ & \\
\hline Non-TNBC & $1(20 \%)$ & $2(40 \%)$ & 0.500 & $2(40 \%)$ & $3(60 \%)$ & 0.500 \\
\hline \multicolumn{7}{|l|}{ E-cadherin } \\
\hline Positive-conversion & $5(100 \%)$ & $0(0 \%)$ & & $2(40 \%)$ & $2(40 \%)$ & \\
\hline Non-positive-conversion & $0(0 \%)$ & $5(100 \%)$ & 0.004 & $3(60 \%)$ & $3(60 \%)$ & 0.738 \\
\hline \multicolumn{7}{|l|}{$\mathrm{N}$-cadherin } \\
\hline Negative-conversion & $3(60 \%)$ & $0(0 \%)$ & $2(40 \%)$ & & $0(0 \%)$ & \\
\hline Non-negative-conversion & $2(40 \%)$ & $5(100 \%)$ & 0.083 & $3(60 \%)$ & $5(100 \%)$ & 0.222 \\
\hline \multicolumn{7}{|l|}{ Vimentin } \\
\hline Negative-conversion & $3(60 \%)$ & $0(0 \%)$ & & $1(20 \%)$ & $2(40 \%)$ & \\
\hline Non-negative-conversion & $2(40 \%)$ & $5(100 \%)$ & 0.083 & $4(80 \%)$ & $3(60 \%)$ & 0.500 \\
\hline \multicolumn{7}{|l|}{ CA9 } \\
\hline Negative-conversion & $4(80 \%)$ & $0(0 \%)$ & & $1(20 \%)$ & $0(0 \%)$ & \\
\hline Non-negative-conversion & $1(20 \%)$ & $5(100 \%)$ & 0.024 & $4(80 \%)$ & $5(100 \%)$ & 0.500 \\
\hline
\end{tabular}

HR: Hormone receptor; HER2: human epidermal growth factor receptor 2; TNBC: triple-negative breast cancer; CA9: carbonic anhydrase 9. 

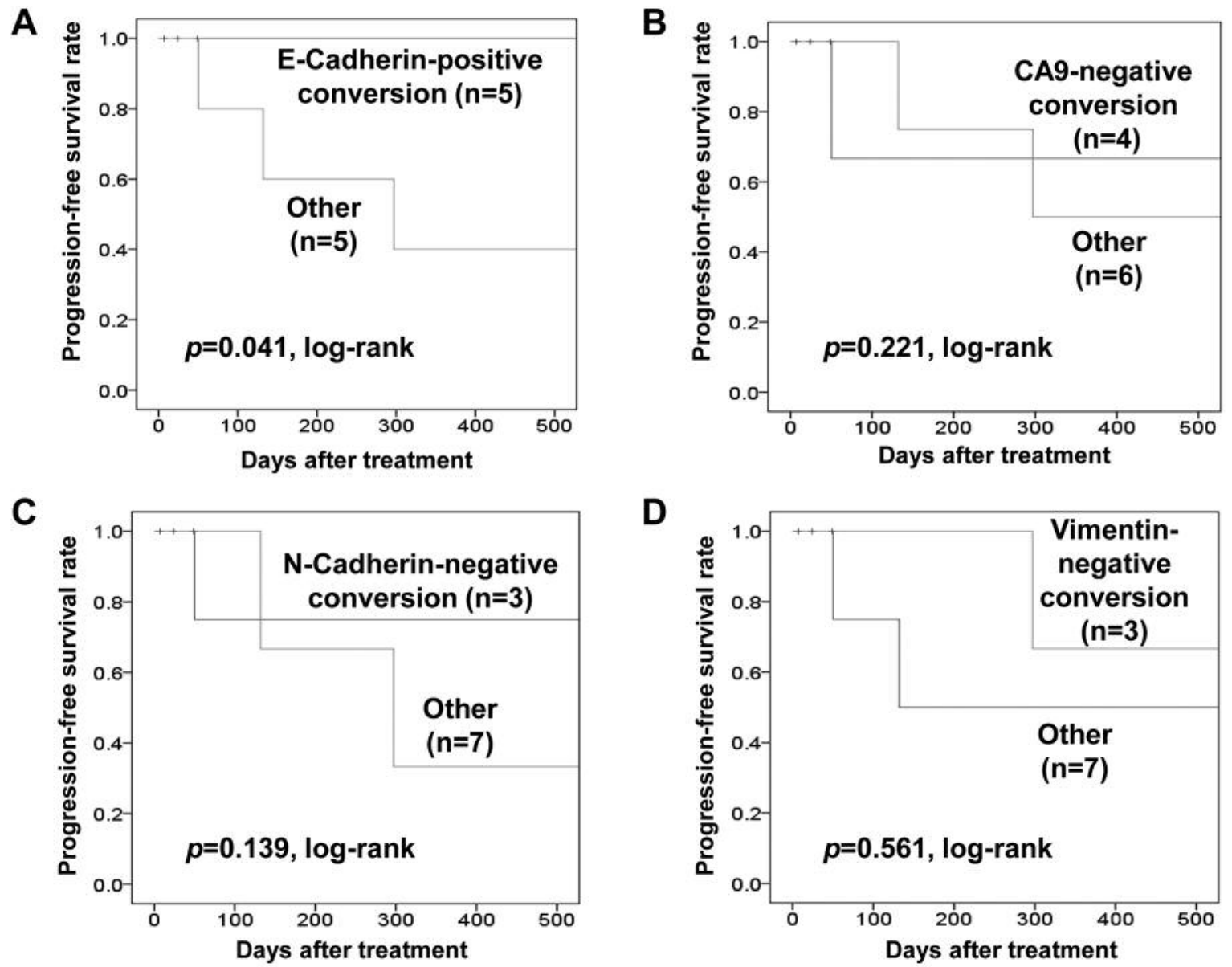

Figure 4. Progression-free survival of eribulin-treated patients with investigated samples. Better progression-free survival (PFS) was observed in patients with tumors that showed E-cadherin-positive conversion $(p=0.041, \log$-rank) (A). No difference in PFS related to carbonic anhydrase 9 (CA9), $N$-cadherin or vimentin expression was observed (B-D).

to treatment (23-25). Thus, suppression of EMT is expected to become a novel anticancer strategy. The expression of EMT markers was evaluated before and after eribulin treatment in clinical samples from patients with breast cancer. Increased E-cadherin expression, and decreased $\mathrm{N}$ cadherin and vimentin expression, which indicate mesenchymal-epithelial transition (MET), were clearly observed in cancer tissues after successful eribulin treatment. In contrast, these observations were not found in tumor tissue of eribulin nonresponders or paclitaxel-treated control patients. Moreover, N-cadherin and vimentin expression decreased in patients with increased E-cadherin expression. Therefore, the 'cadherin switch' was confirmed in the MET. These changes suggest that MET may be a common and unique feature of successful eribulin treatment.

EMT marker expression was compared in tissue samples collected after different treatment protocols in order to distinguish the effects of eribulin treatment and control for the influence of cancer cell heterogeneity within tumors. Another concern was that the cancer cells observed after treatment might be a population that did not reflect the cytotoxic effects of the anticancer drugs. Nevertheless, our observations suggest that cancer cells with a mesenchymal phenotype might have been successfully eradicated by eribulin. Therefore, our results suggest that inhibition of EMT in breast cancer tissues is a major effect of eribulin, which is similar to observations reported in a preclinical study (7).

In experimental animal models of human breast cancer, eribulin was shown to induce microvessel formation in the center of tumor tissues, thereby increasing perfusion within the tumor $(26,27)$. In this study, a significant decrease in CA9 expression was observed in tissues from eribulin responders, indicating a decrease in hypoxia. Hypoxia is considered a key trigger of EMT in cancer cells. Decreased hypoxic stress in cancer tissue may contribute to inhibiting the development of EMT. Remodeling of the vasculature 
A

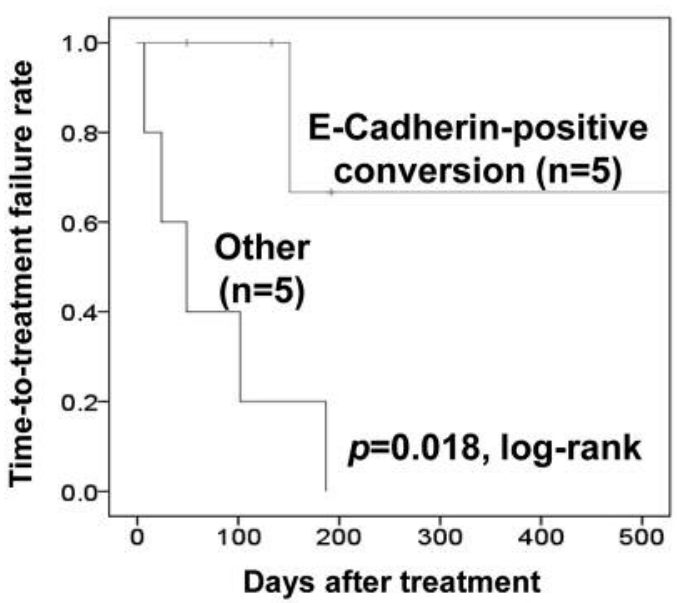

C

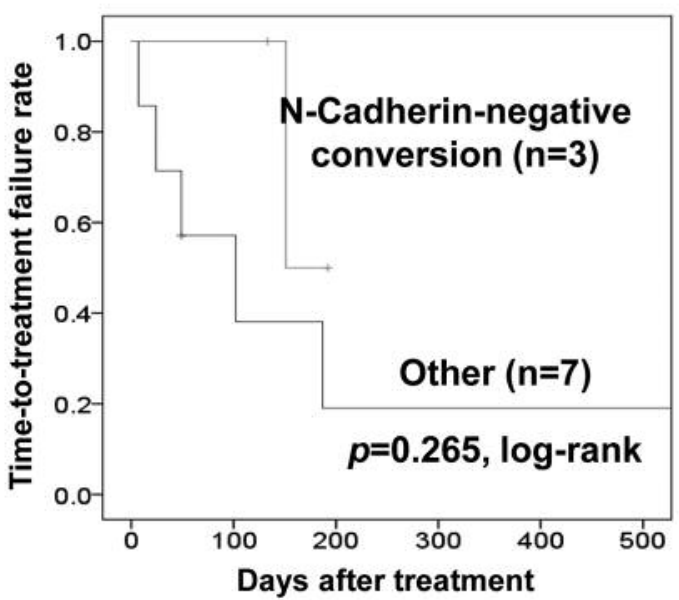

B

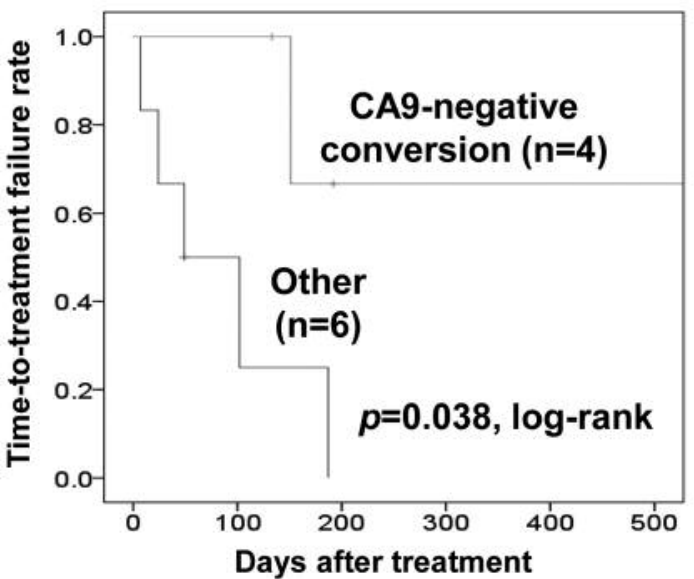

D

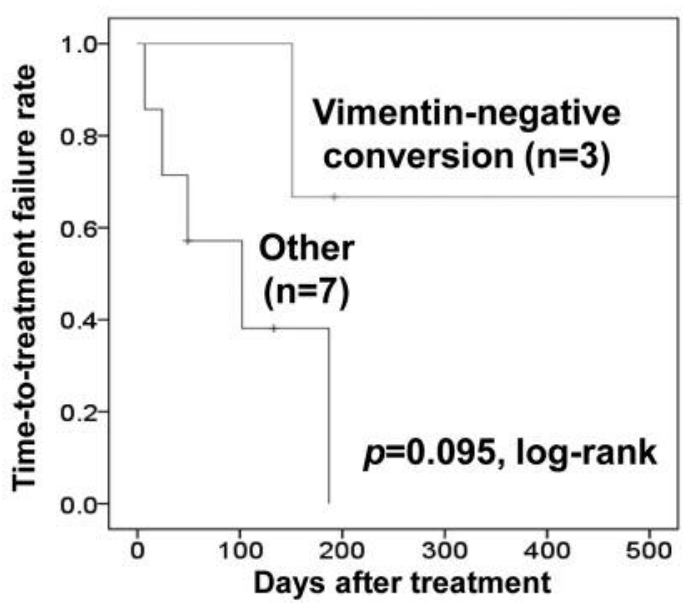

Figure 5. Time-to-treatment failure (TTF) in eribulin-treated patients with investigated samples. Significantly longer TTF was achieved in patients with tumors showing E-cadherin-positive conversion ( $p=0.018$, log-rank test) (A) or carbonic anhydrase 9 (CA9)-negative conversion ( $p=0.038$, log-rank test) (B). No difference was observed in TTF of patients in terms of $N$-cadherin $(C)$ or vimentin (D) expression.

also ensures sufficient drug delivery, thus supporting treatment utility. Furthermore, disappearance of hypoxia contributes to the suppression of angiogenesis-stimulating signals involved in cancer cell proliferation (28).

This study is clinical verification of eriburin chemotherapy using the sample before and after the treatment. Previous studies have not accomplished very much to date. However, the fact that this study was a small-scale retrospective study is a limitation. The expression of EMT and hypoxia markers was evaluated in clinical specimens of breast cancer tissue before and after eribulin therapy. We observed an increase in the expression of E-cadherin, which was concurrent with reduced expression of $\mathrm{N}$-cadherin, vimentin, and CA9 in tissues from patients who responded to eribulin therapy. These results are consistent with observations reported by in vitro studies and in animal models.
Our results suggest that eribulin may act, at least in part, by inhibition of EMT and induction of vascular remodeling. This was a small retrospective study with a non-standardized patient series, but the findings support further investigation of the unique functions of eribulin in prospective studies with larger numbers of participants.

\section{Conflicts of Interest}

The Authors have no conflicts of interest to disclose.

\section{Acknowledgements}

The Authors would like to thank Yayoi Matsukiyo and Tomomi Ohkawa (Department of Surgical Oncology, Osaka City University Graduate School of Medicine) for their helpful advice regarding 
A

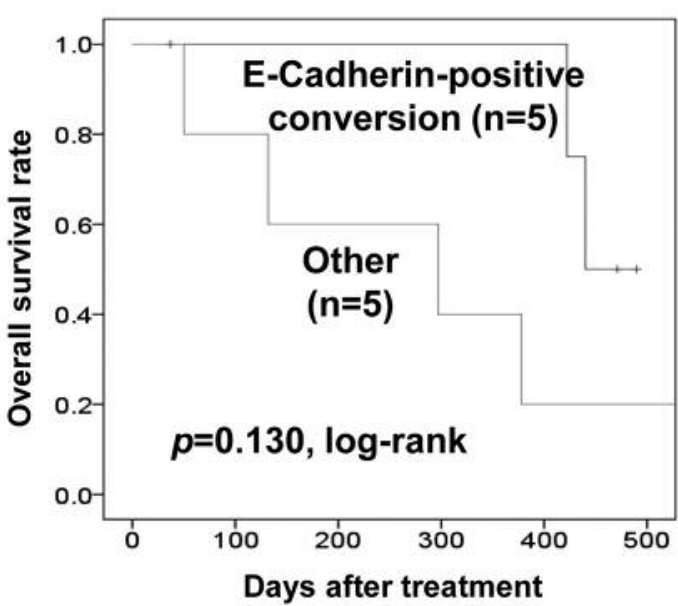

C

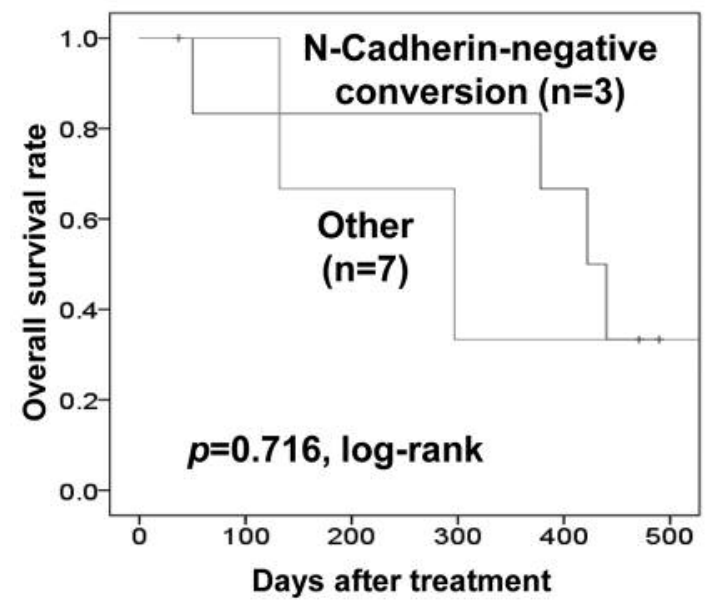

B

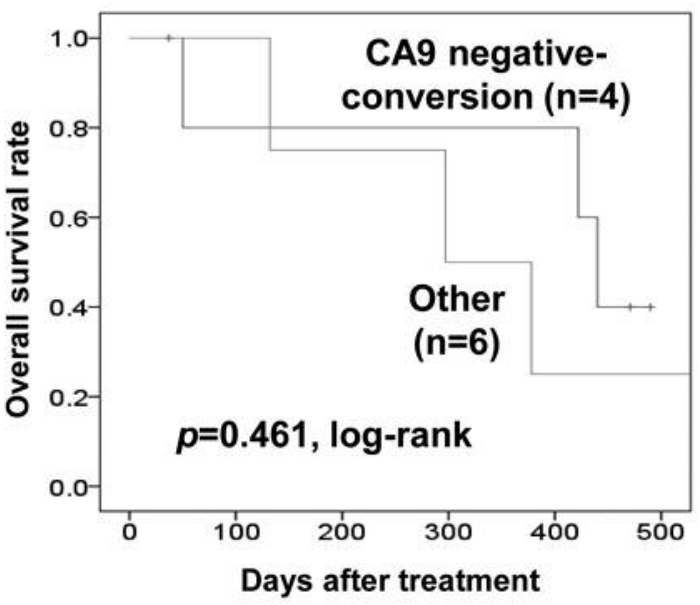

D

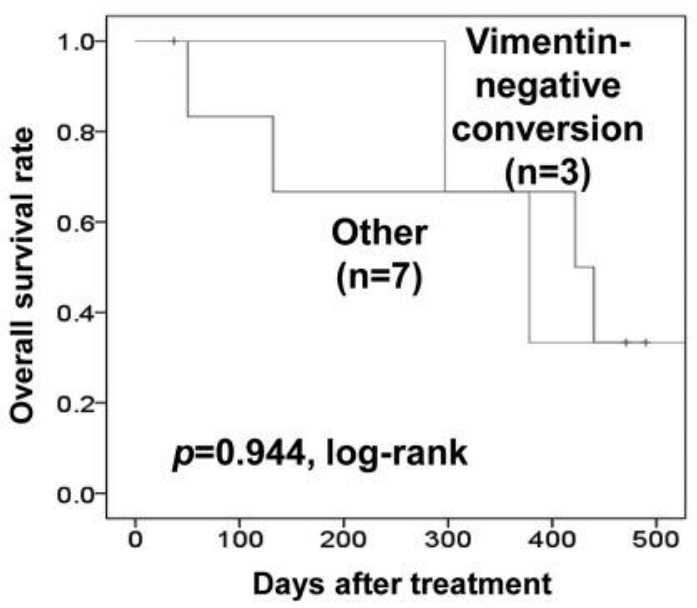

Figure 6. Overall survival (OS) of eribulin-treated patients with investigated samples. There were no differences in OS of patients when stratified by expression or changes of markers of epithelial-mesenchymal transition and carbonic anhydrase 9 (CA9).

data management. This study was funded by grants from the Japan Society for the Promotion of Science (KAKENHI, Nos. 25461992, 26461957, and 17K10559) to Shinichiro Kashiwagi.

\section{References}

1 Shetty N and Gupta S: Eribulin drug review. South Asian J Cancer 3: 57-59, 2014.

2 Jordan MA and Wilson L: Microtubules as a target for anticancer drugs. Nat Rev Cancer 4: 253-265, 2004.

3 Kuznetsov G, Towle MJ, Cheng H, Kawamura T, TenDyke K, Liu D, Kishi Y, Yu MJ and Littlefield BA: Induction of morphological and biochemical apoptosis following prolonged mitotic blockage by halichondrin B macrocyclic ketone analog E7389. Cancer Res 64: 5760-5766, 2004.

4 Bai RL, Paull KD, Herald CL, Malspeis L, Pettit GR and Hamel E: Halichondrin B and homohalichondrin B, marine natural products binding in the vinca domain of tubulin. Discovery of tubulin-based mechanism of action by analysis of differential cytotoxicity data. J Biol Chem 266: 15882-15889, 1991.
5 Ledford H: Complex synthesis yields breast-cancer therapy. Nature 468: 608-609, 2010.

6 Cortes J, O'Shaughnessy J, Loesch D, Blum JL, Vahdat LT, Petrakova K, Chollet P, Manikas A, Dieras V, Delozier T, Vladimirov V, Cardoso F, Koh H, Bougnoux P, Dutcus CE, Seegobin S, Mir D, Meneses N, Wanders J, Twelves $\mathrm{C}$ and investigators E: Eribulin monotherapy versus treatment of physician's choice in patients with metastatic breast cancer (EMBRACE): a phase 3 open-label randomised study. Lancet 377: 914-923, 2011.

7 Yoshida T, Ozawa Y, Kimura T, Sato Y, Kuznetsov G, Xu S, Uesugi M, Agoulnik S, Taylor N, Funahashi Y and Matsui J: Eribulin mesilate suppresses experimental metastasis of breast cancer cells by reversing phenotype from epithelialmesenchymal transition (EMT) to mesenchymal-epithelial transition (MET) states. Br J Cancer 110: 1497-1505, 2014.

8 Dezso Z, Oestreicher J, Weaver A, Santiago S, Agoulnik S, Chow J, Oda Y and Funahashi Y: Gene expression profiling reveals epithelial mesenchymal transition (EMT) genes can selectively differentiate eribulin-sensitive breast cancer cells. PLoS One 9: e106131, 2014. 
9 Mani SA, Guo W, Liao MJ, Eaton EN, Ayyanan A, Zhou AY, Brooks M, Reinhard F, Zhang CC, Shipitsin M, Campbell LL, Polyak K, Brisken C, Yang J and Weinberg RA: The epithelialmesenchymal transition generates cells with properties of stem cells. Cell 133: 704-715, 2008.

10 Morel AP, Lievre M, Thomas C, Hinkal G, Ansieau S and Puisieux A: Generation of breast cancer stem cells through epithelial-mesenchymal transition. PLoS One 3: e2888, 2008.

11 Kajiyama H, Shibata K, Terauchi M, Yamashita M, Ino K, Nawa A and Kikkawa F: Chemoresistance to paclitaxel induces epithelial-mesenchymal transition and enhances metastatic potential for epithelial ovarian carcinoma cells. Int J Oncol 31: 277-283, 2007.

12 Jeanes A, Gottardi CJ and Yap AS: Cadherins and cancer: How does cadherin dysfunction promote tumor progression? Oncogene 27: 6920-6929, 2008.

13 Kokkinos MI, Wafai R, Wong MK, Newgreen DF, Thompson EW and Waltham M: Vimentin and epithelial-mesenchymal transition in human breast cancer - observations in vitro and in vivo. Cells Tissues Organs 185: 191-203, 2007.

14 Sato M, Shames DS and Hasegawa Y: Emerging evidence of epithelial-to-mesenchymal transition in lung carcinogenesis. Respirology 17: 1048-1059, 2012.

15 Dery MA, Michaud MD and Richard DE: Hypoxia-inducible factor 1: regulation by hypoxic and non-hypoxic activators. Int J Biochem Cell Biol 37: 535-540, 2005.

16 Yang AD, Camp ER, Fan F, Shen L, Gray MJ, Liu W, Somcio R, Bauer TW, Wu Y, Hicklin DJ and Ellis LM: Vascular endothelial growth factor receptor-1 activation mediates epithelial to mesenchymal transition in human pancreatic carcinoma cells. Cancer Res 66: 46-51, 2006.

17 Russell J, Carlin S, Burke SA, Wen B, Yang KM and Ling CC: Immunohistochemical detection of changes in tumor hypoxia. Int J Radiat Oncol Biol Phys 73: 1177-1186, 2009.

18 Ivanov S, Liao SY, Ivanova A, Danilkovitch-Miagkova A, Tarasova N, Weirich G, Merrill MJ, Proescholdt MA, Oldfield EH, Lee J, Zavada J, Waheed A, Sly W, Lerman MI and Stanbridge EJ: Expression of hypoxia-inducible cell-surface transmembrane carbonic anhydrases in human cancer. Am J Pathol 158: 905-919, 2001.

19 Kashiwagi S, Yashiro M, Takashima T, Aomatsu N, Kawajiri H, Ogawa Y, Onoda N, Ishikawa T, Wakasa K and Hirakawa K: cKIT expression as a prognostic molecular marker in patients with basal-like breast cancer. Br J Surg 100: 490-496, 2013.

20 Kashiwagi S, Yashiro M, Takashima T, Aomatsu N, Ikeda K, Ogawa Y, Ishikawa T and Hirakawa K: Advantages of adjuvant chemotherapy for patients with triple-negative breast cancer at stage II: usefulness of prognostic markers E-cadherin and Ki67. Breast Cancer Res 13: R122, 2011.
21 Fuyuhiro Y, Yashiro M, Noda S, Kashiwagi S, Matsuoka J, Doi Y, Kato Y, Kubo N, Ohira M and Hirakawa K: Clinical significance of vimentin-positive gastric cancer cells. Anticancer Res 30: 5239-5243, 2010.

22 Aomatsu N, Yashiro M, Kashiwagi S, Kawajiri H, Takashima T, Ohsawa M, Wakasa K and Hirakawa K: Carbonic anhydrase 9 is associated with chemosensitivity and prognosis in breast cancer patients treated with taxane and anthracycline. BMC Cancer 14: 400, 2014.

23 Thomas PA, Kirschmann DA, Cerhan JR, Folberg R, Seftor EA, Sellers TA and Hendrix MJ: Association between keratin and vimentin expression, malignant phenotype and survival in postmenopausal breast cancer patients. Clin Cancer Res 5: 26982703, 1999.

24 Bates RC and Mercurio AM: The epithelial-mesenchymal transition (EMT) and colorectal cancer progression. Cancer Biol Ther 4: 365-370, 2005.

25 Ahmed N, Abubaker K, Findlay J and Quinn M: Epithelial mesenchymal transition and cancer stem cell-like phenotypes facilitate chemoresistance in recurrent ovarian cancer. Curr Cancer Drug Targets 10: 268-278, 2010.

26 Funahashi Y, Okamoto K, Adachi Y, Semba T, Uesugi M, Ozawa Y, Tohyama O, Uehara T, Kimura T, Watanabe H, Asano M, Kawano S, Tizon X, McCracken PJ, Matsui J, Aoshima K, Nomoto $\mathrm{K}$ and Oda $\mathrm{Y}$ : Eribulin mesylate reduces tumor microenvironment abnormality by vascular remodeling in preclinical human breast cancer models. Cancer Sci 105: 13341342, 2014.

27 Agoulnik SI, Kawano S, Taylor N, Oestreicher J, Matsui J, Chow J, Oda Y and Funahashi Y: Eribulin mesylate exerts specific gene expression changes in pericytes and shortens pericyte-driven capillary network in vitro. Vasc Cell 6: 3, 2014.

28 Yancopoulos GD, Davis S, Gale NW, Rudge JS, Wiegand SJ and Holash J: Vascular-specific growth factors and blood vessel formation. Nature 407: 242-248, 2000. 